DOI

\title{
ПРОГНОСТИЧЕСКОЕ ЗНАЧЕНИЕ АНЕМИЧЕСКОГО СИНДРОМА У ПЛАНОВЫХ ХИРУРГИЧЕСКИХ ПАЦИЕНТОВ (ОБЗОР ЛИТЕРАТУРЫ)
}

\section{PROGNOSTIC VALUE OF ANEMIC SYNDROME IN SURGICAL PATIENTS WITH ELECTIVE SURGERY (REVIEW OF LITERATURE)}

\author{
Т.Ю. Калюта ${ }^{1}$, О.А. Кажекин ${ }^{1}$, Т.А. Илясова ${ }^{2}$, О.В. Коченкова ${ }^{3}$ \\ T.Y. Kalyuta ${ }^{1}$, O.A. Kazhekin ${ }^{1}$, T.A. Ilyasova ${ }^{2}$, O.V. Kochenkova ${ }^{3}$ \\ ${ }^{1}$ Филиал частного учреждения образовательной организации высшего образования \\ «Медицинский университет «Реавиз» в городе Саратов, \\ Россия, 410012, г. Саратов, ул. Верхний Рынок, 10 \\ ${ }^{2}$ Клиническая больница имени С.Р. Миротворцева Саратовского Государственного Медицинского \\ Университета имени В.И. Разумовского, Россия, 410054, г. Саратов, ул. Б. Садовая, 137 \\ ${ }^{3}$ ГУЗ «Областная клиническая больница», \\ Россия, 410053, г. Саратов, Смирновское ущелье МКР, Здание 1 \\ ${ }^{1}$ Branch of private institution of educational organization of higher education \\ «Reaviz Medical University» in the city of Saratov, 10 Upper Market St., Saratov, 410012, Russia \\ ${ }^{2}$ Clinical hospital of S.R. Mirotvortsev of the Saratov State Medical University of V.I. Razumovsky \\ 137 Bolshaya Sadovaya St., Saratov, 410054, Russia, \\ ${ }^{3}$ State Health Institution «Regional Clinical Hospital», \\ 1 Smirnovsky gorge of MDT, Saratov, 410053, Russia \\ E-mail: tatianakaluta@yandex.ru
}

\begin{abstract}
Аннотация
Анемический синдром является распространённым предсуществующим явлением среди пациентов, которые поступают в хирургические стационары с целью проведения планового хирургического вмешательства. Общая распространенность её в популяции увеличивается с возрастом, и в пожилом возрасте (>65 лет), по данным ВОЗ, в среднем достигает $11 \%$ и $10,2 \%$ для мужчин и женщин соответственно. Таким образом, ранее недиагностированная анемия часто встречается у плановых хирургических пациентов. Отрицательное прогностическое значение анемии для таких пациентов показано в большом числе зарубежных и ряде отечественных исследований. Данный обзор посвящён влиянию анемии на прогноз при плановых операциях у пациентов различного хирургического профиля. В обзоре также рассмотрено современное состояние вопроса коррекции анемии и влияния гемотрансфузий на прогноз у таких пациентов. Обсуждается международный консенсус и рекомендации по внедрению стратегии «управления кровью пациентов» в хирургическую практику, что позволит снизить затраты и улучшить результаты хирургического вмешательства.
\end{abstract}

\begin{abstract}
Anemic syndrome is a common pre-existing phenomenon among patients who are admitted to surgical hospitals for the purpose of elective surgery. The overall prevalence of it in the population increases with age, and in the elderly ( $>65$ years), according to the data of World Health Organization, on average, reaches $11 \%$ and $10.2 \%$ for men and women, respectively. Thus, previously undiagnosed anemia is often found in patients who undergo elective surgery. Negative prognostic value of anemia for such patients is shown in a large number of foreign and a number of Russian studies. This review focuses on the effect of anemia on the prognosis of planned operations in patients with various surgical profiles. The review also considers the current state of the issue of correction of anemia and the effect of blood transfusions on the prognosis in these patients. The international consensus and recommendations for implementing the
\end{abstract}


«patient blood management» strategy in surgical practice are discussed, which will reduce costs and improve the results of surgical intervention.

Ключевые слова: анемия, анемический синдром, хирургическое вмешательство, прогноз хирургических пациентов, гемотрансфузия.

Keywords: anemia, anemic syndrome, surgical intervention, prognosis of surgical patients, blood transfusion.

\section{Введение}

Общая распространенность анемии в популяции увеличивается с возрастом, и в пожилом возрасте (>65 лет) распространенность анемии, определяемой по критериям Всемирной организацией здравоохранения (ВОЗ), составляет 11 \% и 10,2 \% для мужчин и женщин соответственно [BО3, 1968; Guralnik et al., 2004]. Критерием анемии по ВОЗ служит уровень гемоглобина ниже 12,5 г/дл в качестве нормативной величины как для взрослых мужчин, так и для женщин. Таким образом, ранее недиагностированная анемия часто встречается у плановых хирургических пациентов; ее распространенность зависит от возраста и сопутствующих заболеваний, таких как диабет, застойная сердечная недостаточность и другие заболевания [Кравчун и др., 2013; Goodnough et al., 2003]. По некоторым данным, предоперационная анемия встречается у трети пациентов, перенесших плановую операцию [Clevenger et al., 2015]. Анемия часто встречается в хирургической практике в различных отделениях [Clevenger B. et al., 2015]. Так, по данным D. Kendoff и соавторов, в отделении плановой ортопедической хирургии у $30 \%$ пациентов уровень гемоглобина при поступлении ниже 130 г/л [Kendoff et al., 2011].

L.T. Goodnough и соавторы сообщают, что ранее недиагностированная анемия часто встречается у плановых ортопедических хирургических пациентов в США [Bisbe Vives, 2015].

У европейских пациентов, перенесших плановую ортопедическую операцию, распространенность анемии нарастает с предоперационного уровня в 14,1 \% до 85,8 \% после операции. Среднее снижение гемоглобина составяет при этом 19 г/л и 30 г/л у дооперационно анемичных и неанемичных пациентов соответственно $(\mathrm{P}<0,001)$. Не указывается, с чем связано такое диспропорциональное снижение уровня гемоглобина. Возможно, это связано с предшествующими операциями гемотрансфузиями у анемичных пацинетов. Так, в США 55 \% трансфузий эритроцитарной массы (в общем объёме 6,6 млн единиц в год) назначаются пациентам, которым проводится плановая хирургическая операция [Napolitano, 2005].

В различных хирургических центрах, в зависимости от их уровня и локализации, существенно варьирует процент пациентов с анемией - 8,0 до 18,5 \% [Lasocki et al., 2015]. В одном немецком исследовании была определена большая группа пациентов, подготовленных к плановой операции. Среди таких пациентов обоего пола примерно 29 \% имели анемию [Ellermann et al., 2018]. По сравнению с пациентами, которым планируется большое хирургическое вмешательство, пациенты с планируемым малым хирургическим вмешательством низкого риска имеют минимальную частоту анемии при поступлении - около $0,8 \%$ [Olson et al., 2005].

Отдельную категорию составляют пациенты с онкологической патологией [Muñoz, et al, 2018]. В исследовании, включавшем пациентов, перенесших эзофагэктомию по поводу рака, в целом у 47,6 \% пациентов до операции была выявлена анемия, а у тех, кто получал комбинированное лечение по поводу основного заболевания, частота анемии была выше (60,6 \% против 30,7 \%, P < 0,001) [Melis, et al., 2009].

Таким образом, частота предсуществующей анемии у пациентов, готовящихся к плановым хирургическим вмешательствам, различна у пациентов с различными основны- 
ми заболеваниями, приводящими к необходимости хирургического вмешательства. Наиболее высок процент лиц с анемией среди пациентов с онкологическими процессами.

\section{Этиология анемии}

У пациентов, готовящихся к плановой операции по поводу каого-либо хронического заболевания, анемия традиционно ассоциируется с наличием хронического заболевания и коморбидной патологией [Clevenger et al., 2015]. Открытия в области путей метаболизма железа показали, что хронические заболевания могут вызывать состояние функциональной недостаточности железа, приводящее к анемии [Clevenger et al., 2015]. Ключевой железорегуляторный белок гепсидин, активируемый в ответ на воспаление, ингибирует всасывание железа из желудочно-кишечного тракта и дополнительно снижает биодоступность запасов железа для производства эритроцитов [Clevenger et al., 2015]. Следовательно, хотя запасы железа (преимущественно ферритина) могут быть нормальными, транспорт железа либо из желудочно-кишечного тракта, либо из запасов железа в костный мозг ингибируется, что приводит к состоянию «функционального» дефицита железа и последующей анемии [Clevenger et al., 2015]. Поскольку всасывание из желудочно-кишечного тракта блокируется, увеличение перорального потребления железа неэффективно, и в настоящее время изучается роль внутривенного железа для лечения анемии в условиях хирургических стационаров [Clevenger et al., 2015]. Функциональный дефицит железа (ограничение железа вследствие повышенного уровня гепсидина) является наиболее частой причиной предоперационной анемии [Clevenger et al., 2015]. Другие факторы, вносящие существенный вклад в развитие хронической анемии, не связанной с кровопотерей, определяются некоторыми авторами термином «гематогенная недостаточность» [Kulier et al., 2007]. Распространенность гематогенной недостаточности у пациентов с плановыми операциями составляет, по данным некоторых авторов, 33 \% по уровню железа, 12,3\% по уровню витамина В12 и 3 \% по фолату [Kulier et al., 2007]. Аналогичные данные получены исследователями из Египта и Шотландии [LaPar et al., 2018]. Среди других часто встречающихся факторов, провоцирующих анемию у данной категории пациентов, часто называют хроническую болезнь почек (ХБП) [Guralnik et al., 2004].

Таким образом, анемия у пациентов плановых хирургических отделений любого профиля по этиологии часто относится к «анемии хронического заболевания», что определяет этиотропные подходы к терапии у таких пациентов.

\section{Прогностическое значение анемии при хирургических вмешательствах}

Анализ данных из больших реестров в настоящее время даёт возможность предположить, что анемия является независимым фактором риска, связанным с плохим исходом как в кардиохирургии, так и при других видах хирургических вмешательств [Clevenger et al., 2015]. Пред- и послеоперационная анемия и возникающая в результате этого повышенная потребность в переливании крови являются независимыми факторами риска развития послеоперационной инфекции, более длительного пребывания в стационаре и смертельного исхода [Kansagra et al., 2016; Napolitano L.M., 2005; Kendoff et al., 2011]. Предоперационная анемия связана с худшими исходами и является предиктором послеоперационных переливаний эритроцитов [Kansagra et al., 2016; Napolitano, 2005]. Предоперационная анемия, кровопотеря во время операции и переливание аллогенной крови связаны с повышением послеоперационной заболеваемости и смертности, а также с длительным пребыванием в стационаре пациентов, которым проводятся так называемые «большие хирургические вмешательства» [Muñoz et al., 2016; Desai et al., 2018].

Влияние предоперационной анемии на периоперационную смертность можно проиллюстрировать на примере пациентов, относящихся к Свидетелям Иеговы, которые отказываются от аллогенной трансфузии по религиозным причинам. В ретроспективном исследовании 1958 таких пациентов, перенесших хирургические операции (из анализа ис- 
ключались пациенты, перенёсшие операции на сердце), предоперационная концентрация гемоглобина менее 100 г/л была связана со значительным увеличением периоперационной смертности [Carson et al., 1996]. У пациентов, не получавших предоперационных трансфузий, была установлена связь между самым низким дооперационным уровнем гемоглобина и внутрибольничной кардиальной и некардиальной заболеваемостью и смертностью, а также влияние сопутствующих факторов риска, включённых в оценку по шкале EuroSCORE, на этот эффект. У пациентов с баллом ниже 4 по шкале EuroSCORE отмечается повышение риска некардиальных осложнений, а у пациентов с баллом выше «4» по шкале EuroSCORE и уровнем гемоглобина $<110$ г/л отмечается повышенная частота всех послеоперационных неблагоприятных событий [Kulier et al., 2007]. Низкий предоперационный гемоглобин является независимым предиктором некардиальных («почечных» и «церебральных») неблагоприятных исходов, тогда как увеличение сердечных событий обусловлено другими факторами, связанными с предоперационной анемией [Kulier et al., 2007].

В другом исследовании у всех пациентов послеоперационный уровень гемоглобина ниже 70 г/л ассоциируется с повышением числа осложнений; при этом снижение уровня гемоглобина при уровне ниже 70 г/л увеличивает риск смерти в 1,5 раза [Carson et al., 2002].

У пациентов, перенесших плановую ортопедическую операцию в Европе, послеоперационные осложнения были более частыми у предоперационно анемичных по сравнению с неанемичными пациентами (36,9 против 22,2 \%; P=0,009) [Lasocki et al., 2015].

У кардиохирургических пациентов предоперационная анемия ассоциируется с четырёхкратным увеличением вероятности переливания эритроцитарной массы, увеличением риска почечной недостаточности в 3 раза и с почти удвоением смертности [LaPar et al., 2018].

Данные о влиянии анемии на прогноз у пациентов, которым проводится плановое аортокоронарное шунтирование, получены на большом регистре из 182599 пациентов [Williams et al., 2013]. Это исследование выявило связь между предоперационным гематокритом и исходами операции аортокоронарного шунтирования (АКШ) [Ellermann et al., 2018]. Общая операционная смертность в этом реестре составила 1,6\% [Williams et al., 2013]. По сравнению с пациентами с анемией, определяемой в данном исследовании по уровню гематокрита ниже $33 \%$, пациенты с гематокритом более $42 \%$ имели более низкую смертность $(1,1 \%$ против $3,4 \% ; \mathrm{p}<0,0001)$ и более низкие показатели почечной недостаточности $(2,0 \%$ против $7,8 \%$; $<<0,0001)$, инсульта $(0,9 \%$ против $1,8 \% ; \mathrm{p}<0,0001)$, длительной вентиляции легких $(8,4 \%$ против $17,5 \% ; \mathrm{p}<0,0001)$ и глубокой раневой инфекции грудины (0,3\% против 0,6 \%; p<0,0001) [Williams et al., 2013]. Аналогичные результаты наблюдались и у пациентов, перенесших плановое АКШ, в другом исследовании ( $\mathrm{n}=74$ 292) [Loor et al., 2013]. В этих двух крупных исследованиях [Williams et al., 2013; Loor et al., 2013] процент пациентов с АКШ, получавших периоперационное переливание крови, составлял 88,5 \% в анемической группе (гематокрит при поступлении ниже $33 \%$ ) и составлял 32,5\% у пациентов с гематокритом более $42 \%$ (p<0,0001) [Williams et al., 2013; Loor et al., 2013]. Таким образом, данные двух крупных исследований показывают, что предоперационный уровень гематокрита является мощным независимым предиктором периоперационной смертности, почечной недостаточности, глубокой раневой инфекции грудины у пациентов, перенесших первичные операции АКШ [Williams et al., 2013; Loor et al., 2013].

Отдельно рассматривается группа онкологических пациентов [Новик, 2009]. В исследовании, включавшем пациентов, перенесших эзофагэктомию по поводу рака пищевода, те пациенты, кто получил комбинированное лечение (операция плюс неоадъювантное лечение), были более склонны к анемии (60,6 \% против $30,7 \%, \mathrm{P}<0,001)$. Анемическим больным требовалось больше переливаний крови, чем неанемическим $(46,7 \%$ против $29,6$ \%, P $<0,001)$. Пациенты с любыми периоперационными осложнениями и инфекциями 
хирургического участка чаще получали переливание крови по сравнению с пациентами без осложнений $(\mathrm{OR}=1,73 ; 95$ \% Ди 1,04-2,87 и $\mathrm{OR}=2,98 ; 95$ \% Ди 1,04-8,55 соответственно) [Melis et al., 2009].

\section{Коррекция анемии и её влияние на прогноз}

Несмотря на доказанную роль анемии в плохом прогнозе хирургического вмешательства, она часто недооценивается. Например, исследование, проведенное в Испании, показало, что, хотя предоперационная оценка проводилась в подавляющем большинстве больниц, оптимизация концентрации гемоглобина была предпринята менее чем у $40 \%$ пациентов, которые могли бы извлечь из нее пользу, несмотря на то, что до операции было достаточно времени [Bisbe et al., 2017]. Недооценка анемии обсуждается рядом авторов [Goodnough et al., 2003].

У пациентов, перенесших плановую ортопедическую операцию в Европе, периоперационная коррекция анемии (в основном трансфузионная) была проведена 34,3 \% пациентов. Интраоперационно 14,8 \% предоперационно анемичных и 2,8 \% неанемичных ортопедических пациентов получали трансфузии [Lasocki et al., 2015]. В США 55 \% трансфузий эритроцитарной массы (в общем объёме 6,6 млн единиц в год) назначаются пациентам, которым проводится плановая хирургическая операция [Napolitano, 2005].

Результаты, полученные в одном исследовании по влиянию гематокрита на исход после планового аортокоронарного шунтирования (АКШ), свидетельствуют о том, что не только более низкий предоперационний гематокрит ассоциируется с более высокими показателями периоперационной заболеваемости, но и лечение интраоперационным переливанием крови отрицательно влияет на все группы риска пациентов [Spiegelstein et al., 2015].

Имеются данные, что послеоперационная анемия связана с неблагоприятными сердечно-сосудистыми событиями. Эпизоды периоперационной ишемии миокарда при мониторировании ЭКГ у пациентов, перенесших радикальную простатэктомию, были связаны как с частотой сердечных сокращений, так и с уровнем гематокрита - $28 \%$ [Hogue et al., 1998].

Предоперационная анемия и периоперационное переливание крови являются как идентифицируемыми, так и предотвратимыми хирургическими рисками [Clevenger et al., 2015].

Лечение анемии исторически было сосредоточено на использовании переливания крови в качестве решения проблемы анемии в периоперационном периоде. Но теперь данные больших исследований показывают, что переливание крови, по-видимому, не улучшает риски у пациентов, а фактически может увеличить риск послеоперационных осложнений и длительность пребывания в больнице [Clevenger et al., 2015]. Так, по данным некоторых авторов, не только анемия, но и факт переливания крови, независимо от наличия анемии, связаны с неблагоприятными исходами операции [Clevenger et al., 2015]. Это подтверждается и данными других авторов, которые отмечают, что трансфузия эритроцитарной массы ассоциируется с повышенной послеоперационной смертностью (отношение шансов [OR], 4,3; $\mathrm{P}<0.0001)$ [LaPar et al., 2018]. Аналогичные результаты были продемонстрированы в исследовании Ad N. и соавторов, где хорошо установлена связь между более низким предоперационным гематокритом и риском заболеваемости/смертности после кардиохирургических операций: в этом исследовании переливание крови было единственным прогностическим фактором для послеоперационного появления осложнений как у женщин $(\mathrm{OR}=4,56, \mathrm{P}<0,001)$, так и у мужчин (OR=9,22, $\mathrm{P}<0,001)$ [Ad et al., 2015]. Pocсийские авторы также утверждают, что проведение гемотрансфузии не только сопровождается большими затратамии, но и может привести к серьезным осложнениям [Моисеев, 2013]. 
Гемотрансфузии - это эффективный метод лечения угрожающей жизни анемии, позволяющий быстро увеличить уровни гемоглобина и гематокрита, однако они дают кратковременный эффект и имеют серьезные недостатки (высокая стоимость, возможный дефицит препаратов крови, осложнения) [Рогачевский и др., 2014].

Таким образом, результаты и зарубежных, и отечественных исследований показывают, что, хотя воздействие анемии или переливания эритроцитарной массы сами по себе ассоциированы с более высоким риском для хирургических пациентов, однако эти риски ниже, чем неблагоприятное влияние сочетания анемии и переливания эритроцитарной массы [Loor et al., 2013, Рогачевский и др., 2014].

При этом рядом авторов подчёркивается, что неблагоприятное независимое влияние трансфузий не является поводом для отказа от коррекции анемии, а наоборот, является поводом для поиска новых путей коррекции предоперационной анемии. Большинство авторов сходятся во мнении, что анемию следует рассматривать как серьезное и поддающееся лечению заболевание, а не просто аномальное лабораторное значение [Goodnough et al., 2011]. В большинстве случаев анемия может быть скорректирована до плановой операции, а предоперационные вмешательства, направленные на коррекцию анемии, должны уменьшить при этом количество послеоперационных трансфузий и улучшить исходы. Показано, что лечение периоперационной анемии снижает потребность в трансфузии эритроцитарной массы и улучшает исход заболевания [Kansagra et al., 2016; Napolitano, 2005].

В немецком исследовании, где перед плановой операцией рассчётный риск переливания эритроцитарной массы составлял более $10 \%$, лечение анемии до операции с внутривенным введением железа было связано со снижением частоты переливания эритроцитарной массы (итсследование проводилось на группе гинекологических и акушерских пациентов) [Ellermann et al., 2018].

В России проведен ряд исследований по оценке эффективности препаратов железа при коррекции анемии в ходе подготовки пациентов к обширным плановым оперативным вмешательствам [Горохова и др., 2013; Моисеев, 2013; Моисеев, 2012; Рогачевский и др., 2014].

Таким образом, на настоящее время накоплено достаточно данных для того, чтобы утверждать необходиомость активных подходов к коррекции предоперационной анемии, с учётом выявленного отрицательного влияния предоперационных гемотрансфузий на прогноз. В этих пилотных исследованиях было показано, что введение большой дозы железа позволяет сократить необходимое число инфузий и добиться увеличения уровня гемоглобина за короткий срок, что имеет особое значение в предоперационном периоде [Моисеев, 2012].

\section{Заключение}

Ряд опытных исследователей и клиницистов приняли участие в экспертном семинаре и разработали следующее консенсусное заявление - «международное консенсусное заявление по периоперационному лечению анемии и дефицита железа», опубликованное в 2017 году [Muñoz, et al., 2017]. Основные положения, озвученные в данном консенсусном заявлении, и подтверждённые представленными выше исследованиями, представлены ниже:

1. Доказано, что периоперационная анемия связана с повышением заболеваемости и смертности у хирургических больных.

2. Значение предоперационной анемии представляется недооцененным, и ее выявление должно привести к плановому обследованию и лечению перед плановой операцией. В большинстве случаев анемия может быть исправлена до проведения плановых операций и вмешательств.

3. Все чаще признается опасность ненужного переливания крови.

4. Рекомендуется стратегическое внедрение стратегии «управления кровью пациентов» в хирургическую практику, что позволит снизить затраты и улучшить результаты хи- 
рургического вмешательства [Clevenger et al., 2015]. Предоперационная оптимизация ведения пациентов, которым планируются хирургические вмешательства, потенциально связанные со значительной кровопотерей наряду со стратегиями минимизации интраоперационной кровопотери, показывают перспективность снижения послеоперационных трансфузий и улучшения исходов.

5. Будущие исследования должны оценить сроки и методы оптимизации предоперационной анемии в хирургии и определить, какие пациенты являются лучшими кандидатами для терапии [Kansagra et al., 2016].

В целом, было обнаружено мало пользы от применения внутривенного железа для лечения предоперационной анемии. В лучшем случае, было обнаружено, что прием препаратов железа снижает долю пациентов, нуждающихся в переливании крови, и число переливаемых единиц в наблюдательных исследованиях в ортопедической хирургии, но не в кардиохирургии. Для пациентов, перенесших ортопедические операции, у которых ожидается развитие тяжелой послеоперационной анемии, предлагается внутривенное введение железа в пред- и периоперационном периоде (рекомендация, основанная на доказательствах категории «С»). Для всех других видов хирургии не может быть сделано никаких научно обоснованных рекомендаций. Группа рекомендует провести крупные проспективные РКИ для оценки эффективности и безопасности внутривенного введения железа хирургическим пациентам [Beris, 2008]. Одно из таких исследований было проведено в России, и оно показало эффективность затрат на лечение предоперационной анемии внутривенными препаратами железа [Горохова и др., 2013].

В настоящее время считается, что краеугольными камнями подхода «управления кровью пациентов в хирургии» является тройственный подход: надлежащее лечение анемии, предотвращение кровопотери и ограничительное переливание крови [Butcher et al., 2018].

В консенсусном заявлении подчёркивается, что необходимо провести крупномасштабные проспективные рандомизированные клинические исследования по вмешательствам по поводу предоперационной анемии, которые могут наиболее эффективно решить важные клинические вопросы и эту явно неудовлетворенную медицинскую потребность [Muñoz, et al, 2018].

\section{Список литературы}

1. Горохова С.Г., Ряженов В.В., Емченко И.В. 2013. Фармакоэкономическая оценка эффективности препаратов железа при коррекции анемии в ходе подготовки пациентов к обширным плановым оперативным вмешательствам. Клинфармакол тер. 22 (3): 47-52.

2. Кравчун П.Г., Ефремова О.А., Рындина Н.Г., Титова А.Ю. 2013. Структура анемического синдрома и особенности трофологического статуса у больных с хронической сердечной недостаточностью и хронической болезнью почек. Научные ведомости Белгородского государственного университета. Серия: Медицина. Фармация. 11 (154): 102-106.

3. Моисеев С.В. 2012. Железа карбоксимальтозат (Феринжект) - новый внутривенный препарат для лечения железодефицитной анемии. Клин фармакол. тер. 21 (2): 48-53.

4. Моисеев С.В. 2013. Влияние внутривенных препаратов железа на потребность в гемотрансфузиях при анемии. Клин. фармакол. тер. 22 (5): 60-65.

5. Новик А.В. 2019. Анемия и метаболические расстройства у онкологических больных. Практическая онкология. 10 (3): 131-40.

6. Рогачевский О.В., Моисеев С.В. 2014. Клиническое значение и лечение анемии в предоперационном периоде. Клиническая фармакология и терапия. 23 (2): 55-60.

7. Ad N., Holmes S.D., Massimiano P.S., Spiegelstein D., Shuman D.J., Pritchard G., Halpin L. 2015. Operative risk and preoperative hematocrit in bypass graft surgery: Role of gender and blood transfusion. Cardiovasc. Revasc. Med. Oct.-Nov.;16 (7): 397-400.

8. Beris P., Muñoz M., García-Erce J.A., Thomas D., Maniatis A., Van der Linden P. 2008. Perioperative anaemia management: consensus statement on the role of intravenous iron. Br. J. Anaesth. 100 (5): 599-604. 
9. Bisbe E., Basora M., Colomina M.J. 2017. Peri-operative treatment of anaemia in major orthopaedic surgery: a practical approach from Spain. Spanish Best Practice in Peri-operative Anaemia Optimisation Panel. Blood. Transfus. 15 (4): 296-306.

10. Bisbe Vives E. 2015. Treatment of preoperative anemia in major orthopedic surgery. Rev. Esp. Anestesiol. Reanim. 62 (1): 52-6.

11. Butcher A., Richards T. 2018. Cornerstones of patient blood management in surgery. Transfus. Med. 28 (2): 150-157.

12. Carson J.L., Duff A., Poses R.M., Berlin J.A., Spence R.K., Trout R., Noveck H., Strom B.L. 1996. Effect of anaemia and cardiovascular disease on surgical mortality and morbidity. Lancet; 348: $1055-60$.

13. Carson J.L., Noveck H., Berlin J.A., Gould S.A. 2002. Mortality and morbidity in patients with very low postoperative $\mathrm{Hb}$ levels who decline blood transfusion. Transfusion. 42: 812-8.

14. Clevenger B., Richards T. 2015. Pre-operative anaemia. Anaesthesia. 2015 Jan; 70 Suppl. $1: 20-8, \mathrm{e} 6-8$.

15. Clevenger B., Mallett S.V., Klein A.A., Richards T. 2015. Patient blood management to reduce surgical risk. Br. J. Surg. 102 (11): 1325-37.

16. Desai N., Schofield N., Richards T. 2018. Perioperative Patient Blood Management to Improve Outcomes. Anesth Analg. 127 (5): 1211-1220.

17. Ellermann I., Bueckmann A., Eveslage M., Buddendick H., Latal T., Niehoff D., Geissler R.G., Hempel G., Kerkhoff A., Berdel W.E., Roeder N., Van Aken H.K., Zarbock A., Steinbicker A.U. 2018. Treating Anemia in the Preanesthesia Assessment Clinic: Results of a Retrospective Evaluation. Anesth. Analg. 127 (5): 1202-1210.

18. Goodnough L.T., Maniatis A., Earnshaw P., Benoni G., Beris P., Bisbe E., Fergusson D.A., Gombotz H., Habler O., Monk T.G., Ozier Y., Slappendel R., Szpalski M. 2011. Detection, evaluation, and management of preoperative anaemia in the elective orthopaedic surgical patient: NATA guidelines. Br. J. Anaesth. 106 (1): 13-22.

19. Goodnough L.T., Nissenson A.R., Dubois R.W. 2003. Anemia: not just an innocent bystander? Arch Intern Med, 163: 1400-4.

20. Guralnik J.M., Eisenstaedt R.S., Ferrucci L., Klein H.G., Woodman R.C. 2004. Prevalence of anemia in persons 65 years and older in the United States: evidence for a high rate of unexplained anemia. Blood, 104: 2263-8.

21. Hogue C.W. Jr., Goodnough L.T., Monk T.G. 1998. Perioperative myocardial ischemic episodes are related to hematocrit level in patients undergoing radical prostatectomy. Transfusion. 38: 924-31.

22. Kansagra A.J., Stefan M.S. 2016. Preoperative Anemia: Evaluation and Treatment. Anesthesiol Clin. 34 (1): 127-41.

23. Kansagra A.J., Stefan M.S. 2016. Preoperative Anemia: Evaluation and Treatment. Anesthesiol Clin. 34 (1): 127-41.

24. Kendoff D., Tomeczkowski J., Fritze J., Gombotz H., von Heymann C. 2011. Preoperative anemia in orthopedic surgery: clinical impact, diagnostics and treatment. Orthopade. 40 (11): 1018-20, $1023-5,1027-8$.

25. Kulier A., Levin J., Moser R., Rumpold-Seitlinger G., Tudor I.C., Snyder-Ramos S.A., Moehnle P., Mangano D.T. 2007. Impact of preoperative anemia on outcome in patients undergoing coronary artery bypass graft surgery. Investigators of the Multicenter Study of Perioperative Ischemia Research Group; Ischemia Research and Education Foundation. Circulation. 31;116 (5): 471-9.

26. LaPar D.J., Hawkins R.B., McMurry T.L., Isbell J.M., Rich J.B., Speir A.M., Quader M.A., Kron I.L., Kern J.A., Ailawadi G. 2018. Preoperative anemia versus blood transfusion: Which is the culprit for worse outcomes in cardiac surgery? Investigators for the Virginia Cardiac Services Quality Initiative. J. Thorac. Cardiovasc. Surg. 156 (1): 66-74.

27. Lasocki S., Krauspe R., von Heymann C., Mezzacasa A., Chainey S., Spahn D.R. 2015. PREPARE: the prevalence of perioperative anaemia and need for patient blood management in elective orthopaedic surgery: a multicentre, observational study. Eur. J. Anaesthesiol. 32 (3): 160-7.

28. Loor G., Rajeswaran J., Li L., Sabik J.F., Blackstone E.H., McCrae K.R., Koch C.G. 2013. The least of 3 evils: exposure to red blood cell transfusion, anemia, or both? J. Thorac. Cardiovasc. Surg. 146 (6): 1480-1487. 
29. Melis M., McLoughlin J.M., Dean E.M., Siegel E.M., Weber J.M., Shah N., Kelley S.T., Karl R.C. 2009. Correlations between neoadjuvant treatment, anemia, and perioperative complications in patients undergoing esophagectomy for cancer. J. Surg. Res. 1:153 (1): 114-20.

30. Muñoz M., Acheson A.G., Auerbach M., Besser M., Habler O., Kehlet H., Liumbruno G.M., Lasocki S., Meybohm P., Rao Baikady R., Richards T., Shander A., So-Osman C., Spahn D.R., Klein A.A. 2017. International consensus statement on the peri-operative management of anaemia and iron deficiency. Anaesthesia. 72 (2): 233-247.

31. Muñoz M., Acheson A.G., Bisbe E., Butcher A., Gómez-Ramírez S., Khalafallah A.A., Kehlet H., Kietaibl S., Liumbruno G.M., Meybohm P., Rao Baikady R., Shander A., So-Osman C., Spahn D.R., Klein A.A. 2018. An international consensus statement on the management of postoperative anaemia after major surgical procedures. Anaesthesia. 73 (11): 1 418-1431.

32. Muñoz M., Gómez-Ramírez S., Kozek-Langeneker S. 2016. Pre-operative haematological assessment in patients scheduled for major surgery. Anaesthesia. 71 (1): 19-28.

33. Napolitano L.M. 2005. Perioperative anemia. Surg. Clin. North. Am. 85 (6): 1215-27.

34. Nutritional anaemias. Report of a WHO scientific group. World Health Organization Technical Report Series No. 405. Geneva: World Health Organization, 1968.

35. Olson R.P., Stone A., Lubarsky D. 2005. The prevalence and significance of low preoperative hemoglobin in ASA 1 or 2 outpatient surgery candidates. Anesth. Analg. 101 (5): 1337-40.

36. Spiegelstein D., Holmes S.D., Pritchard G., Halpin L., Ad N. 2015. Preoperative hematocrit as a predictor of perioperative morbidities following nonemergent coronary artery bypass surgery. $\mathrm{J}$. Card. Surg. 30 (1): 20-6.

37. Williams M.L., He X., Rankin J.S., Slaughter M.S., Gammie J.S. 2013. Preoperative hematocrit is a powerful predictor of adverse outcomes in coronary artery bypass graft surgery: a report from the Society of Thoracic Surgeons Adult Cardiac Surgery Database. Ann Thorac Surg. 96 (5): 1628-34.

\section{References}

1. Gorokhova S.G., Ryazhenov V.V., Emchenko I.V. 2013. Pharmacoeconomical evaluation of the effectiveness of iron preparations for the correction of anemia in the course of preparing patients for extensive planned surgical interventions. Conformal Ter., 22 (3): 47-52.

2. Kravchun P.G., Efremova O.A., Ryndina N.G., Titova A.Ju. 2013. Struktura anemicheskogo sindroma i osobennosti trofologicheskogo statusa u bol'nyh s hronicheskoj serdechnoj nedostatochnost'ju i hronicheskoj bolezn'ju pochek [Structure of anemic syndrome and features of trophological status in patients with chronic heart failure and chronic kidney disease]. Nauchnye vedomosti Belgorodskogo gosudarstvennogo universiteta. Serija: Medicina. Farmacija. 11 (154): 102-106.

3. Moiseev S.V. 2012. Iron carboxymaltose (Ferinject) - a new intravenous drug for the treatment of iron deficiency anemia. Klin Pharmacol Ter. 21 (2): 48-53.

4. Moiseev S.V. 2013. Effect of intravenous iron preparations on the need for blood transfusions in anemia. Klin Pharmacol Ter. 22 (5): 60-65.

5. Novik A.V. 2019. Anemia and metabolic disorders in cancer patients. Practical Oncology.10 (3): 131-40.

6. Rogachevsky O.V., Moiseev S.V. 2014. Clinical significance and treatment of anemia in the preoperative period. Clinical pharmacology and therapy. 23 (2): 55-60.

7. $\quad$ Ad N., Holmes S.D., Massimiano P.S., Spiegelstein D., Shuman D.J., Pritchard G., Halpin L. 2015. Operative risk and preoperative hematocrit in bypass graft surgery: Role of gender and blood transfusion. Cardiovasc. Revasc. Med. Oct.-Nov.;16 (7): 397-400.

8. $\quad$ Beris P., Muñoz M., García-Erce J.A., Thomas D., Maniatis A., Van der Linden P. 2008. Perioperative anaemia management: consensus statement on the role of intravenous iron. Br. J. Anaesth. 100 (5): 599-604.

9. Bisbe E., Basora M., Colomina M.J. 2017. Peri-operative treatment of anaemia in major orthopaedic surgery: a practical approach from Spain. Spanish Best Practice in Peri-operative Anaemia Optimisation Panel. Blood. Transfus. 15 (4): 296-306.

10. Bisbe Vives E. 2015. Treatment of preoperative anemia in major orthopedic surgery. Rev. Esp. Anestesiol. Reanim. 62 (1): 52-6.

11. Butcher A., Richards T. 2018. Cornerstones of patient blood management in surgery. Transfus. Med. 28 (2): 150-157. 
12. Carson J.L., Duff A., Poses R.M., Berlin J.A., Spence R.K., Trout R., Noveck H., Strom B.L. 1996. Effect of anaemia and cardiovascular disease on surgical mortality and morbidity. Lancet; 348: 1055-60.

13. Carson J.L., Noveck H., Berlin J.A., Gould S.A. 2002. Mortality and morbidity in patients with very low postoperative $\mathrm{Hb}$ levels who decline blood transfusion. Transfusion. 42: 812-8.

14. Clevenger B., Richards T. 2015. Pre-operative anaemia. Anaesthesia. 2015 Jan; 70 Suppl. $1: 20-8$, e6-8.

15. Clevenger B., Mallett S.V., Klein A.A., Richards T. 2015. Patient blood management to reduce surgical risk. Br. J. Surg. 102 (11): 1325-37.

16. Desai N., Schofield N., Richards T. 2018. Perioperative Patient Blood Management to Improve Outcomes. Anesth Analg. 127 (5): 1211-1220.

17. Ellermann I., Bueckmann A., Eveslage M., Buddendick H., Latal T., Niehoff D., Geissler R.G., Hempel G., Kerkhoff A., Berdel W.E., Roeder N., Van Aken H.K., Zarbock A., Steinbicker A.U. 2018. Treating Anemia in the Preanesthesia Assessment Clinic: Results of a Retrospective Evaluation. Anesth. Analg. 127 (5): 1202-1210.

18. Goodnough L.T., Maniatis A., Earnshaw P., Benoni G., Beris P., Bisbe E., Fergusson D.A., Gombotz H., Habler O., Monk T.G., Ozier Y., Slappendel R., Szpalski M. 2011. Detection, evaluation, and management of preoperative anaemia in the elective orthopaedic surgical patient: NATA guidelines. Br. J. Anaesth. 106 (1): 13-22.

19. Goodnough L.T., Nissenson A.R., Dubois R.W. 2003. Anemia: not just an innocent bystander? Arch Intern Med, 163: 1400-4.

20. Guralnik J.M., Eisenstaedt R.S., Ferrucci L., Klein H.G., Woodman R.C. 2004. Prevalence of anemia in persons 65 years and older in the United States: evidence for a high rate of unexplained anemia. Blood, 104: 2263-8.

21. Hogue C.W. Jr., Goodnough L.T., Monk T.G. 1998. Perioperative myocardial ischemic episodes are related to hematocrit level in patients undergoing radical prostatectomy. Transfusion. 38: 924-31.

22. Kansagra A.J., Stefan M.S. 2016. Preoperative Anemia: Evaluation and Treatment. Anesthesiol Clin. 34 (1): 127-41.

23. Kansagra A.J., Stefan M.S. 2016. Preoperative Anemia: Evaluation and Treatment. Anesthesiol Clin. 34 (1): 127-41.

24. Kendoff D., Tomeczkowski J., Fritze J., Gombotz H., von Heymann C. 2011. Preoperative anemia in orthopedic surgery: clinical impact, diagnostics and treatment. Orthopade. 40 (11): 1018-20, $1023-5,1027-8$.

25. Kulier A., Levin J., Moser R., Rumpold-Seitlinger G., Tudor I.C., Snyder-Ramos S.A., Moehnle P., Mangano D.T. 2007. Impact of preoperative anemia on outcome in patients undergoing coronary artery bypass graft surgery. Investigators of the Multicenter Study of Perioperative Ischemia Research Group; Ischemia Research and Education Foundation. Circulation. 31;116 (5): 471-9.

26. LaPar D.J., Hawkins R.B., McMurry T.L., Isbell J.M., Rich J.B., Speir A.M., Quader M.A., Kron I.L., Kern J.A., Ailawadi G. 2018. Preoperative anemia versus blood transfusion: Which is the culprit for worse outcomes in cardiac surgery? Investigators for the Virginia Cardiac Services Quality Initiative. J. Thorac. Cardiovasc. Surg. 156 (1): 66-74.

27. Lasocki S., Krauspe R., von Heymann C., Mezzacasa A., Chainey S., Spahn D.R. 2015. PREPARE: the prevalence of perioperative anaemia and need for patient blood management in elective orthopaedic surgery: a multicentre, observational study. Eur. J. Anaesthesiol. 32 (3): 160-7.

28. Loor G., Rajeswaran J., Li L., Sabik J.F., Blackstone E.H., McCrae K.R., Koch C.G. 2013. The least of 3 evils: exposure to red blood cell transfusion, anemia, or both? J. Thorac. Cardiovasc. Surg. 146 (6): $1480-1487$.

29. Melis M., McLoughlin J.M., Dean E.M., Siegel E.M., Weber J.M., Shah N., Kelley S.T., Karl R.C. 2009. Correlations between neoadjuvant treatment, anemia, and perioperative complications in patients undergoing esophagectomy for cancer. J. Surg. Res. 1:153 (1): 114-20.

30. Muñoz M., Acheson A.G., Auerbach M., Besser M., Habler O., Kehlet H., Liumbruno G.M., Lasocki S., Meybohm P., Rao Baikady R., Richards T., Shander A., So-Osman C., Spahn D.R., Klein A.A. 2017. International consensus statement on the peri-operative management of anaemia and iron deficiency. Anaesthesia. 72 (2): 233-247.

31. Muñoz M., Acheson A.G., Bisbe E., Butcher A., Gómez-Ramírez S., Khalafallah A.A., Kehlet H., Kietaibl S., Liumbruno G.M., Meybohm P., Rao Baikady R., Shander A., So-Osman C., 
Spahn D.R., Klein A.A. 2018. An international consensus statement on the management of postoperative anaemia after major surgical procedures. Anaesthesia. 73 (11): 1 418-1431.

32. Muñoz M., Gómez-Ramírez S., Kozek-Langeneker S. 2016. Pre-operative haematological assessment in patients scheduled for major surgery. Anaesthesia. 71 (1): 19-28.

33. Napolitano L.M. 2005. Perioperative anemia. Surg. Clin. North. Am. 85 (6): 1215-27.

34. Nutritional anaemias. Report of a WHO scientific group. World Health Organization Technical Report Series No. 405. Geneva: World Health Organization, 1968.

35. Olson R.P., Stone A., Lubarsky D. 2005. The prevalence and significance of low preoperative hemoglobin in ASA 1 or 2 outpatient surgery candidates. Anesth. Analg. 101 (5): 1337-40.

36. Spiegelstein D., Holmes S.D., Pritchard G., Halpin L., Ad N. 2015. Preoperative hematocrit as a predictor of perioperative morbidities following nonemergent coronary artery bypass surgery. $\mathrm{J}$. Card. Surg. 30 (1): 20-6.

37. Williams M.L., He X., Rankin J.S., Slaughter M.S., Gammie J.S. 2013. Preoperative hematocrit is a powerful predictor of adverse outcomes in coronary artery bypass graft surgery: a report from the Society of Thoracic Surgeons Adult Cardiac Surgery Database. Ann Thorac Surg. 96 (5): 1628-34.

\section{Ссылка для цитирования статьи For citation}

Калюта Т.Ю., Масляков В.В., Кажекин О.А., Илясова Т.А., Коченкова О.В. 2020. Прогностическое значение анемического синдрома у плановых хирургических пациентов (обзор литературы). Актуальные проблемы медицины, 43(1): 165-175. DOI

Kalyuta T.Y., Maslyakov V.V., Kazhekin O.A., Ilyasova T.A., Kochenkova O.V. 2020. Prognostic value of anemic syndrome in surgical patients with elective surgery (review of literature). Challenges in Modern Medicine, 43(1): 165-175 (in Russian). DOI 